Bundesgesundheitsbl $2011 \cdot 54: 401-403$

DOI 10.1007/s00103-011-1241-1

Online publiziert: 4. April 2011

(c) Springer-Verlag 2011

\author{
U. Koch ${ }^{1}$ F. Petermann ${ }^{2}$ \\ ${ }^{1}$ Institut für Medizinische Psychologie, Universitätsklinikum \\ Hamburg-Eppendorf, Hamburg \\ ${ }^{2}$ Lehrstuhl für Klinische Psychologie und Diagnostik, Zentrum für Klinische \\ Psychologie und Rehabilitation der Universität Bremen, Bremen
}

\title{
Innovationen in der medizinischen Rehabilitation
}

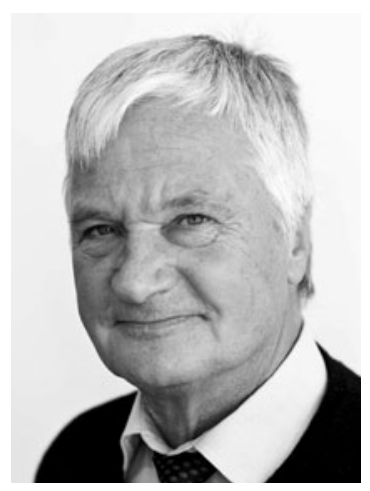

litationsmaßnahmen darauf, Menschen mit chronischen Krankheiten und Behinderungen dazu zu verhelfen, die Erkrankung und ihre Folgen zu bewältigen, um möglichst weitgehend und selbstständig am normalen Leben in Familie, Beruf und Gesellschaft teilnehmen zu können. Für den Fall, dass eine vollständige Rehabilitation nicht $z u$ erreichen ist, sollen die Auswirkungen von Behinderung und chronischer Erkrankung auf die genannten Lebensbereiche auf ein Minimum reduziert werden.

Die häufigsten Indikationen für medizinische Interventionen im Rahmen von Rehabilitationsmaßnahmen in Deutschland sind Erkrankungen des Stütz- und Bewegungsapparates, psychische Erkrankungen und Herz-Kreislauf-Erkrankungen. Als Stärken des in Deutschland bereits vor 120 Jahren etablierten Systems der medizinischen Rehabilitation gelten der große Fundus des in dieser Zeit gesammelten rehabilitativen Wissens und eine moderne Rehabilitationsgesetzgebung, die Rahmenbedingungen und finanzielle Voraussetzungen für die Reha-

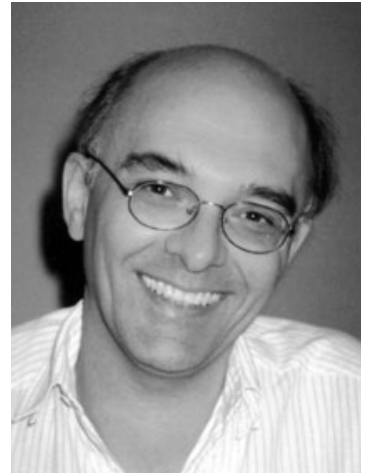

bilitation sicherstellt. Deutschland weist ein flächendeckendes Netz spezialisierter rehabilitativer Einrichtungen auf, in denen ein umfassendes Rehabilitationsverständnis die Grundlage der Maßnahmengestaltung bildet. Eine personelle Ausstattung der Einrichtungen mit interdisziplinär zusammengesetzten Behandlungsteams ist die Regel.

In den letzten 20 Jahren gab es $v$. $a$. in der Folge einer kritischen Bestandsaufnahme der Rehabilitation durch die Reha-Reformkommission des damals noch bestehenden Verbands Deutscher Rentenversicherungsträger zahlreiche und intensive Bemühungen um eine Weiterentwicklung der medizinischen Rehabilitation in Deutschland. Dieser Veränderungsprozess zielte vorrangig auf eine stärkere Bedarfsorientierung, eine Flexibilisierung der Angebote, ergänzende Angebote im Bereich der ambulanten Rehabilitation, die Überwindung von Schnittstellen und eine Intensivierung von Qualitätssicherung und Rehabilitationsforschung. So wurde in den letzten Jahren intensiv an der Entwicklung geeigneter Verfahren zur 
Feststellung des Rehabilitationsbedarfs gearbeitet. Diese Bemühungen richteten sich insbesondere auf die Erfassung der rehabilitationsspezifischen Krankheitsfolgen im Sinne der ICF. Eine stärkere Flexibilisierung bedeutet eine variable, auf die individuellen Problemlagen der Rehabilitandinnen und Rehabilitanden orientierte Gestaltung der Hilfsangebote. Diese kann sich auf die Art und Dichte der rehabilitativen Leistungen, deren zeitliche Verteilung sowie auf die Wahl des Rehabilitationssettings beziehen. Bezüglich der zeitlichen Flexibilisierung von Rehabilitationsmaßnahmen gab es in den letzten Jahren im Rahmen von Modellversuchen eine Reihe von Erprobungen, unter anderem zur Frage der Möglichkeiten einer sog. "Intervallbehandlung“. Auch haben die Kliniken inzwischen einen größeren Handlungsspielraum dahingehend, dass sie im Rahmen einer festgelegten durchschnittlichen Verweildauer für ihre Rehabilitanden die Behandlungsdauer im Einzelfall weitgehend autonom und unabhängig vom Kostenträger festlegen können. Mit der Entwicklung ambulanter rehabilitativer Angebote ist die Hoffnung auf die Realisierung wohnortnaher Hilfsangebote und die bessere Nutzung der lokal gegebenen Hilfsmöglichkeiten und Ressourcen verbunden. Ambulante Rehabilitationsmaßnahmen sollen auch dazu beitragen, bisher unterversorgte Gruppen von rehabilitationsbedürftigen Versicherten zu erreichen. Die großen Rehabilitationsträger haben in den letzten 15 Jahren unterschiedliche Ansätze der ambulanten Rehabilitation entwickelt, in Modellversuchen erprobt und evaluiert und in einzelnen indikativen Bereichen (insbesondere in der orthopädischen Rehabilitation) implementiert.

Die Rehabilitation in Deutschland wird seit Längerem wegen verschiedener ungelöster Schnittstellenprobleme kritisch diskutiert. Diese zeigen sich unter anderem auf der Ebene der unterschiedlich zuständigen Kostenträger, zwischen den verschiedenen Versorgungsbereichen/Institutionen (rehabilitative Versorgung, ambulante und stationäre Akutversorgung) und zwischen den verschiedenen Rehabilitationsbereichen (medizinische und berufliche Rehabilitation). Schnittstellenprobleme sind mit Qualitätseinbu- ßen und vermeidbaren Kosten verbunden, da fehlende Informationsflüsse und Transparenz zu mangelnder Kontinuität und Verzögerungen in der Behandlung sowie zu Mehrfachuntersuchungen und den damit verbundenen Belastungen führen. Diese Probleme betreffen im besonderen Maße Patienten mit chronischen Erkrankungen, da diese mit einem häufig wechselnden und schwer vorhersagbaren Versorgungsbedarf einhergehen. Die zahlreichen in der Vergangenheit praktizierten Lösungsversuche, die Übergänge zwischen den verschiedenen Versorgungsangeboten besser zu regeln, waren insgesamt wenig erfolgreich. Neue Hoffnungen richten sich auf die in der Gesundheitspolitik der letzten Jahre stark favorisierten Disease-Management-Programme und Konzepte der integrierten Versorgung. Die Bemühungen zielen hier auf eine Einbettung der Rehabilitation in die Gesamtversorgung. Unterschiedliche Vorbereitungs- und Nachsorgeangebote wurden entwickelt und in Modellversuchen erfolgreich erprobt. Um der beruflichen Reintegration, dem zentralen Anliegen der medizinischen Rehabilitation, besser gerecht $z u$ werden, wurden in den letzten Jahren auch besondere Bemühungen unternommen, die „Medizinisch-beruflich orientierte Rehabilitation" weiterzuentwickeln und so medizinische und berufliche Rehabilitationsmaßnahmen stärker $z u$ vernetzen.

Die Rentenversicherung und die gesetzlichen Krankenkassen haben in den letzten zehn Jahren erhebliche Anstrengungen unternommen, um die Qualität ihrer Leistungen durch wissenschaftlich fundierte und extern gesteuerte Programme sicherzustellen. Zentrale Instrumente sind dabei die Strukturerhebung, das Peer-Review-Verfahren und die Patientenbefragung. Später kam die Entwicklung von rehabilitationsspezifischen Leitlinien im Sinne von Therapiestandards hinzu. Ebenfalls seit Mitte der 199oer Jahre hat sich in Deutschland eine disziplinübergreifende Rehabilitationswissenschaft an mehreren Universitäten etablieren können. Vor allem dem von 1998 bis 2004 gemeinsam von der Rentenversicherung und dem Bundesforschungsministerium finanzierten rehabilitationswissenschaftlichen Verbundforschungs- programm kommt für die Verbesserung der Rahmenbedingungen der Rehabilitationsforschung besondere Bedeutung zu. Die vorrangig in diesem wie auch in den verschiedenen Nachfolgeprogrammen bearbeiteten Themen waren Entstehungszusammenhänge, Verlauf und Prognose von chronischen Krankheiten, Behinderungen und ihre Folgen, rehabilitationsdiagnostische Verfahren sowie die Weiterentwicklung und Evaluation von Rehabilitationsmaßnahmen. Letztere Forschungsthemen beinhalteten auch die Klärung von Fragen im Hinblick auf den Rehabilitationsbedarf und den Zugang zu Maßnahmen der Rehabilitation einschließlich der Erreichbarkeit von rehabilitativen Versorgungsangeboten sowie die ökonomische Evaluation der rehabilitativen Versorgung. Ein Teil der Ergebnisse des Verbundforschungsprogramms konnte unmittelbar zur Optimierung der Angebote in der medizinischen Rehabilitation genutzt werden.

In den letzten Jahren sind darüber hinaus in der Rehabilitation eine stärkere präventive Orientierung sowie eine stärkere Einforderung der Mitwirkungspflicht und Eigenverantwortung der Rehabilitanden festzustellen. Des Weiteren wurde versucht, durch die Entwicklung von zielgruppenspezifischen Ansätzen, die zum Beispiel das Geschlecht, ein höheres Alter oder einen Migrationshintergrund berücksichtigen, den Zugang und das Ergebnis der Rehabilitation zu verbessern.

Das hier vorgelegte Themenheft stellt mit insgesamt zwölf Beiträgen wichtige aktuelle Weiterentwicklungen der Rehabilitation in Deutschland dar.

Im ersten Artikel beschreiben R. Buschmann-Steinhage und R. Brüggemann den Veränderungsprozess, so wie er sich aus der Perspektive der Deutschen Rentenversicherung darstellt. Sie dokumentieren dies zum einen am Wandel des Rehabilitationsbedarfs, der Inanspruchnahme, des Indikationsspektrums und der Rehabilitationssettings, zum anderen an qualitativen Veränderungen, die sich auf eine stärkere Betonung von Evidenzorientierung, beruflichen Problemlagen in den RehaKonzepten, auf konsequente Vernetzung der Rehabilitation im Gesundheitssystem sowie auf eine stärkere Präventions- und Patientenorientierung beziehen. Der zu- 
letzt genannte Aspekt der Stärkung der Selbstbeteiligung in der Rehabilitation wird thematisch im nachfolgenden Beitrag von J. Dirmaier und M. Härter vertieft. Die Autoren sehen Potenziale zur stärkeren Partizipation auf der Ebene der medizinischen Entscheidungsprozesse, auf der Ebene des Managements der eigenen Erkrankung sowie auf der Systemebene. Sie kritisieren die bisherige unzureichende Umsetzung dieser Konzepte in die Versorgung und analysieren die aus ihrer Sicht hierfür verantwortlichen Barrieren.

M. Morfeld, A. Strahl und U. Koch beschreiben und bilanzieren anschließend die Entwicklung der ambulanten Rehabilitation in Deutschland während der letzten 15 Jahre. Dargestellt werden die gesetzlichen Grundlagen für ambulante Rehabilitation, die Entwicklung der Konzepte, die erreichten Zielgruppen und die zur ambulanten Rehabilitation durchgeführten rehabilitationswissenschaftlichen Untersuchungen. Auf der Basis der aufgezeigten Grenzen der bisherigen Ansätze und Modelle der ambulanten Rehabilitation in Deutschland werden Perspektiven für deren Weiterentwicklung diskutiert.

Im anschließenden Beitrag setzen sich E. Farin, M. Glattacker und W.H. Jäckel mit dem Stand der Entwicklung und der Implementierung von Leitlinien in der medizinischen Rehabilitation auseinander. Sie vertreten die Einschätzung, dass verschiedene inzwischen vorliegende und spezifisch auf den Bereich der Rehabilitation ausgerichtete Leitlinien bezüglich der Evidenzbasis den akutmedizinischen Leitlinien nicht nachstehen. Dennoch sehen sie in Bezug auf die methodischen Gütekriterien einen deutlichen Verbesserungsbedarf. Für die Leitlinienforschung in der Rehabilitation empfehlen die Autoren eine Konzentration auf Spezifika dieses Versorgungsbereichs wie auf die Gestaltung berufsgruppenübergreifender Leitlinien oder auf die Anwendbarkeit von Leitlinien bei multiplen Erkrankungen.

Die berufliche Reintegration ist nach wie vor die primäre Zielsetzung der Rehabilitation der gesetzlichen Rentenversicherung. In dem Beitrag von E. Röckelein, M. Lukasczik und S. Neuderth werden neuere Entwicklungen in der „Medizinisch-beruflich orientierten Rehabilita- tion" vorgestellt. Beschrieben werden das von der Rentenversicherung entwickelte Anforderungsprofil und die Möglichkeiten der Umsetzung solcher Angebote in Diagnostik und Therapie. Im nachfolgenden Beitrag beziehen sich $H$. Faller, A. Reusch und K. Meng auf innovative Schulungskonzepte in der medizinischen Rehabilitation. Diese sollen den Rehabilitandinnen und Rehabilitanden Strategien und Fertigkeiten zur Verfügung stellen, um informierte Entscheidungen treffen und ein gelungenes Selbstmanagement hinsichtlich Gesundheit und Lebensstil vornehmen zu können. Unter didaktischen Gesichtspunkten werden interaktive und teilnehmerorientierte Vorgehensweisen favorisiert.

A. Kobelt, M. Winkler und F. Petermann stellen anschließend am Beispiel der psychosomatischen Rehabilitation Konzepte zur Vorbereitung sowie zur Nachbereitung medizinischer Rehabilitationsmaßnahmen vor. Sie sehen in solchen Ansätzen die Voraussetzung für eine erfolgreiche Wiedereingliederung in das Erwerbsleben nach stationärer medizinischer Rehabilitation. Der Beitrag von H. Kordy, F. Theis, M. Wolf knüpft daran unmittelbar an. Die Autoren beschreiben hier die Potenziale einer Internet-vermittelten Nachsorge für den gleichen rehabilitativen Indikationsbereich.

Die nächsten beiden Beiträge beschreiben die Rehabilitanden mit Migrationshintergrund in der psychosomatischen Rehabilitation: M.-O. Mösko, S. Pradel und H. Schulz zeigen in einer eigenen Untersuchung auf, dass v. a. türkische Rehabilitandinnen und Rehabilitanden und solche aus dem ehemaligem Jugoslawien stärker belastet sind und ein höheres Risiko für einen negativen Behandlungserfolg aufweisen als Rehabilitandinnen und Rehabilitanden ohne Migrationshintergrund. A. Kobelt, J. Göbber und F. Petermann setzen sich mit den kulturell bedingten wie auch mit weiteren Hemmfaktoren in der Rehabilitation von Migrantinnen und Migranten auseinander, die für die schlechteren Behandlungsergebnisse verantwortlich sein könnten. Die Autoren leiten daraus die Notwendigkeit von integrativen und flexibilisierten Formen der psychosomatischen Rehabilitation $a b$.
Die letzten beiden Beiträge beziehen sich einerseits auf ganz junge, andererseits auf ältere Rehabilitanden: F. Petermann und C.-P. Bauer sehen einen deutlich gestiegenen Rehabilitationsbedarf bei Kindern und Jugendlichen. Durch moderne und frühzeitige rehabilitationsspezifische Diagnostik und spezialisierte multimodale Interventionen wollen sie einem Fortschreiten der Chronifizierung der Erkrankung und ihrer Folgen entgegenwirken. W. von Renteln-Kruse, J. Anders und U. Dapp setzen sich abschließend mit der Frage auseinander, wie der Grundsatz "Rehabilitation vor Pflege" realisiert werden kann. Die Autoren geben eine Übersicht zum rationalen Hintergrund, zu prinzipiellen Grundlagen und zur inhaltlichen sowie organisatorischen Konzeption spezifisch geriatrischer Rehabilitation.

Die Herausgeber des Themenheftes wünschen den Leserinnen und Lesern eine von ihnen als sehr interessant erlebte Auseinandersetzung mit dem Thema Rehabilitation!

Ihre

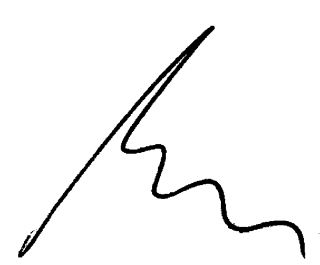

Uwe Koch

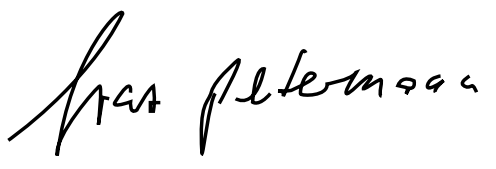

Franz Petermann

\section{Korrespondenzadresse \\ Prof. Dr. U. Koch}

Institut für Medizinische Psychologie, Universitätsklinikum Hamburg-Eppendorf Martinistr. 52, 20246 Hamburg

koch@uke.uni-hamburg.de

\section{Prof. Dr. F. Petermann}

Lehrstuhl für Klinische Psychologie und Diagnostik, Zentrum für Klinische Psychologie und Rehabilitation der Universität Bremen Grazer Straße 6, 28359 Bremen

fpeterm@uni-bremen.de 